\title{
New joint design to create a more natural and efficient biped
}

\author{
Giuseppina Gini*, Umberto Scarfogliero and Michele Folgheraiter \\ Department of Electronics and Information, Politecnico di Milano, Piazza L. da Vinci 32, MILANO, I-20133, Italy
}

(Received 22 February 2008; final version received 8 January 2009)

\begin{abstract}
This paper presents a human-oriented approach to design the mechanical architecture and the joint controller for a biped robot. Starting from the analysis of the human lower limbs, we figured out which features of the human legs are fundamental for a correct walking motion, and can be adopted in the mechanical design of a humanoid robot. We focus here on the knee, designed as a compliant human-like knee instead of a classical pin-joint, and on the foot, characterised by the mobility and lightness of the human foot. We implemented an elastic actuator, with a simple position control paradigm that sets the joint stiffness in real time, and developed the basic controller. Results in simulation are discussed. In our approach the robot gains in adaptability and energetic efficiency, which are the most challenging issues for a biped robot.
\end{abstract}

Keywords: humanoid robotics; bipedal robotics; biomimetic robotics; compliant joint; stiffness controller; gait analysis

\section{Introduction}

The last century witnessed the birth and development of robotics and its impressive progress. In the industry robots are well integrated with the other automatic machines, and they can operate faster with higher precision in comparison to human beings. Nevertheless, if we take a closer look at the kinematic structure of these systems, we can see how they are limited in mobility.

This is more evident if we intend to apply those robots in an unstructured environment, like a home. First, the robot should be able to move around avoiding and surpassing obstacles. These movements should also be performed with compliance to the safety of the human beings that are in the environment. Moreover, the robots should be able to use tools and other machines designed for human use and based on human kinematic abilities.

A possible solution for mobility is the choice of a wheeled traction system. This usually moves in a simple manner on flat floors; it is efficient from the energetic point of view, since the centre of mass (CM) acts on a straight line during the movement. However it presents important limitations, for example it is not possible for such robots to climb over obstacles bigger than their wheel dimensions.

Those limitations can be overcome if the robot is equipped with legs, that normally act by increasing the robot's Degrees of Freedom (DoF). A pioneering contribution in biped robots was done by Lim et al. (2004).

Several other modern robots are designed to walk and behave like humans (Hashimoto and al 2002; Hirai et al. 1998) but until now the efficiency of the human gait is still far from being reached.
In this regard, the work of (McGeer 1990) is remarkable. His passive dynamic walker made a stable gait without close position control, considering the walking motion as a natural oscillation of a double pendulum; and this is actually how humans seem to walk (Kiriazov 1991; Gottlieb et al. 1996). His results inspired many other works, such as the stability analysis (Garcia et al. 1998) and the physical implementation (Wisse et al. 2001; Kuo 1999; Collins et al. 2001) of several prototypes.

In this paper we present Light Adaptive-Reactive biPed (LARP), our humanoid legged system developed at the department of electronics and information of the 'Politecnico di Milano' University, with the aim to explain how the mechanical design makes the robot able to adapt to the real operating environment. Our aim was to create a system that could represent a good model of human lower limbs, in order to understand how a natural walking motion is achieved and how it can be implemented in a humanoid robot. For this reason, we adopted anthropomorphic feet, knees and a mass-distribution similar to the human limbs.

According to McGeer (1990) we designed an actuation system that can take advantage of the natural dynamics of the link. Studying the results of our controller we found several similarities with the assumptions of the equilibrium point theory. This is a widely debated theory, formulated in 1965 by A. Feldman (Asatryan and Feldman 1965; Feldman 1966a; 1966b), and still in evolution. This theory proposes that the segmental reflexes, together with the muscle-skeletal system, behave like a spring. Movement is achieved just by moving the equilibrium position of that spring (Latash and Gottlieb 1991; McIntyre and Bizzi

*Corresponding author. Email: gini@elet.polimi.it 
1993; Gottlieb et al. 1989), and this is actually how our actuator, provided with visco-elastic elements (Scarfogliero et al. 2004), performs the movement.

In the following sections we concentrate on robot design, with particular emphasis on the knee, which presents several similarities to the human articulation, and the foot, developed with two passive DoF. Then we describe the actuators and the controller; we illustrate results on simulating the movement at the joint level. We also present the analysis and comparison of two different kinds of gaits both from the kinematic and the efficiency point of view. The last section outlines the conclusions and proposes future developments.

\section{Bio-mechanical and constructive aspects of biped robots}

If we consider the mass distribution in the human body, we observe that the structure evolved together with biped walking. About $70 \%$ of the mass is in the upper part of the body, while feet are only $10 \%$. A man during a normal activity consumes approximatively $320 \mathrm{~W}$ of power, and about $25 \%$ of it is consumed in working. To compare with robots, the M2 biped (Paluska 2000) from MIT, a robot designed to use the natural dynamics of the links, uses 90 $\mathrm{W}$ for each motor and employs twelve motors to move its $40 \mathrm{~kg}$ of weight.

From the study of the muscle activity, we know that humans, differently from robots, do not activate the muscles in any moment for any position but instead the leg naturally oscillates during walking as a pendulum, requiring muscle activity only to initiate or terminate the motion (McMahon 1984).

Many studies on biomechanics of human walking (Vaughan 2003) are available. Some explain the evolution of biped walking as a trend to improve motion efficiency; others consider how the activity changes during transportation of loads. It has been observed that African women are able to carry over their head a weight as big as $20 \%$ of their body weight without incrementing metabolic activity, perhaps due to a better exchange between potential and kinetic energy, or given to the high $\mathrm{CM}$ which improves stability reducing the efforts to compensate for errors.

Another theory explains walking, as other rhythmic acquired movements, as generated from the central pattern generator (CPG) nervous systems located in the spinal area (Pinter et al. 1998) and able to generate the electric rhythmic pulses necessary to activate the muscles to the needed frequency, without direct involvement of the central nervous system.

Biped robots can be classified according to the capability to perform static or dynamic gait. Static walking assumes that, during walking, the inertial forces derived from accelerations are irrelevant with respect to the robot weight. The static gait is very slow, and the CM is always inside the area covered by the foot. Robots with dynamic walking are faster and move more naturally. Since inertial forces are no longer irrelevant, the position of the CM alone is unable to guarantee the equilibrium of the robot. In this case we should force the projection of the zero moment point (ZMP), the point where all the moments acting on the robot sum to zero to stay inside the area covered by the foot (Surla et al. 1989).

Another classification of bipeds considers the mechanical complexity of the robot and counts the DoFs. A bidimensional biped needs 6 DoFs to walk, while a threedimensional (3D) one is subject to oscillations in the frontal plane (roll) and in the horizontal plane (yaw), arising from the contact with the ground and from the rotation of the free leg, so a compensation is required. The compensation can be given adding a mass, or adding new DoF. In general 3D bipeds have $12 \mathrm{DoFs}$, two in the ankle, one in the knee and three in the hip.

In $1990 \mathrm{McGeer}$ presented an innovative approach (McGeer 1990), designing a biped with passive dynamic walking (PDW), able to use the gravity to walk autonomously on a descending plane. The biped, different from a kinematic chain whose joints positions have to be determined, is a double pendulum, whose natural oscillation brings it in contact with the ground in a stable way, and where gravity and inertia help in the movement. The controller in PDW only has to compensate for disturbance forces.

Most of the available biped robots are very different from the McGeer design. They concentrate on the rotations of joints, modelling walking as a sequence of positions to reach which they obtain by equilibrium monitoring the ZMP. To produce natural walking they generate the sequence in a computer, and keep ZMP inside the foot and in case of disturbances they compute little movements to bring the ZMP back to an optimal position.

\section{The robot knee}

The most obvious function of the knee is lifting the shank for foot clearance.

Using stiff legs could actually simplify the motion and the robot structure (examples go from the simple Fallis's toy (Fallis 1888) to the 3D biped robot of MIT LegLab).

After PDW (McGeer 1990), where McGeer demonstrated how to exploit the mass distribution of the robot to make it walk on a shallow slope without actuation, he added a knee joint in order to have a fully-passive walker (McGeer 1990; Collins et al. 2001; Wisse and Frankenhuyzen 2002). This joint empowers the swinging motion due to gravity, and with the right mass distribution, it is possible to perform a fully-passive pace.

Apart from PDW, the knee is fundamental to ensure energetic efficiency. In a robot with straight legs the foot clearance would have to be created by an additional pelvic tilt. This means a reduced step length and an increased 

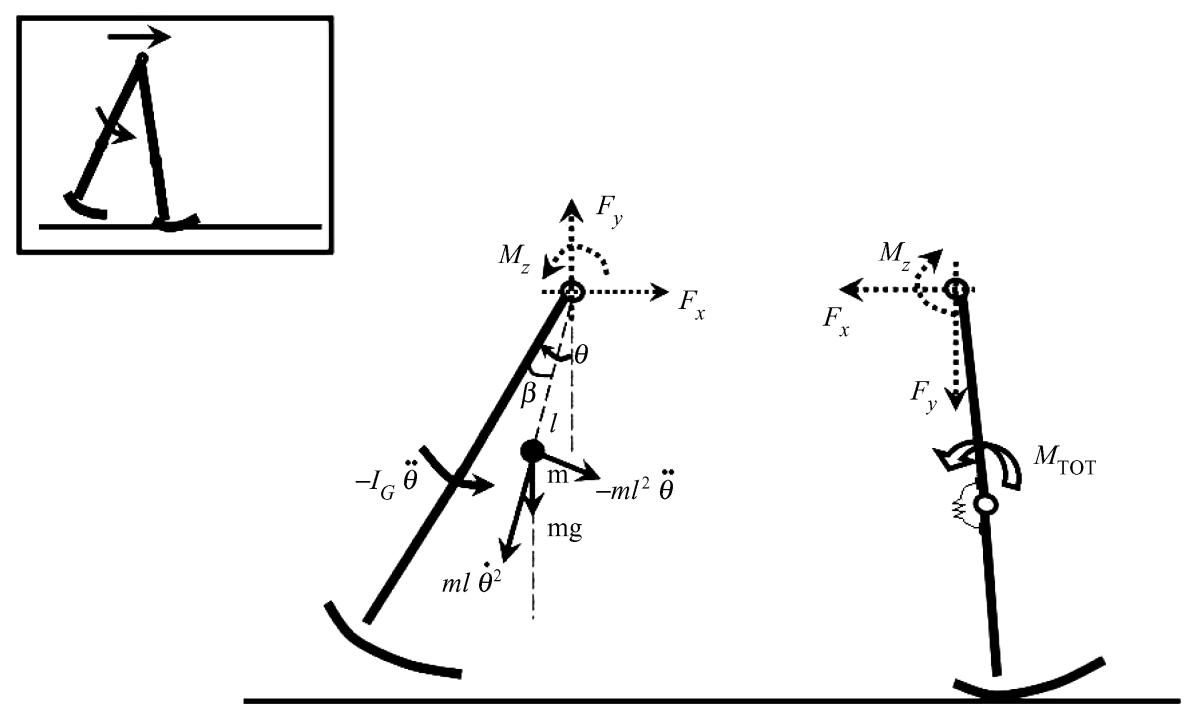

Figure 1. During walking, the knee of the stance leg has to counteract the inertial loads due to the swinging motion. In this case the knee can be exploited to store energy, acting as a spring.

energy consumption, as the pelvis is the heaviest part of the body while the knee just lifts the foot. This has a big influence on walking efficiency (Koopman et al. 2001). Another effect of straight legs would be that the double support time is decreased during gait, making the straight leg walking more critical for stability.

The knee is also important during the stance phase, while the supporting leg remains straight. In this case, while the swinging leg moves forward, the knee of the stance leg has to counteract the inertial load generated by gait motion, as shown in figure 1. Using a force control to actuate the knee we demonstrated (Scarfogliero et al. 2004), that it is possible to store energy and exploit the natural dynamics of the walking motion. The same happens in humans: during the stance phase the knee bends a bit, storing energy as a spring would do. This energy is then released to empower the hip forward motion, with a relevant increase in the step length and foot clearance. The result is a more stable walk with the same energy consumption, as already underlined on the PDW robot Mike (Wisse and Frankenhuyzen 2002).

\subsection{New design of the knee joint}

For the knee the most obvious and adopted solution in robotics is a simple pin joint. The motor is applied directly to the joint, or, for mass-distribution reasons, is placed in the upper part of the robot (Pratt et al. 2001).

Looking at the prosthesis field, we find a completely different approach. Passive prosthesis have to perform the knee bending using inertial torque generated by the forward acceleration of the thigh, in a similar manner as in PDW. In addition, for obvious safety reasons, during the stance phase the knee has to be locked. Today, prosthetic knees are build using multi-axial mechanisms. The characteristic of these mechanisms is that during motion, the centre of rotation ( $c r$ ) is not fixed, as in a pin joint, but moves along a trajectory that depends on the mechanism structure. As the stability of the knee during the stance phase strongly depends on the $\mathrm{cr}$ position, varying the mechanism proportions, it is possible to have different $c r$ trajectories with different stability properties.

Human articulation can be represented by two rolling surfaces kept together by different ligaments. A fundamental role is covered by the anterior and posterior cruciate ligaments that prevent the posterior and anterior displacement of the tibia relative to the femur. This structure is very close to the compliant rolling-contact joint designed by J. Herder (Jeanneau et al. 2004). This joint is composed of two circular surfaces rolling on each other. Flexible bands constrain the joint, leaving only one degree of freedom, i.e. the rotation along the joint axis. During the motion, the tendons wrap on a surface or on the other, letting the joint rotate without scratch. This significantly reduces friction.

Critical in this joint are the torsional stiffness and the rigidity respective to external forces. This issue is fundamental for the robot, where the knee is subject to high torsional and flexional disturbances. In our design we strengthened the joint, producing the articulation shown in Figure 2. Instead of flexible bands, we used three Coramide strings that can all be independently fastened. Furthermore a special track mechanism avoids lateral sliding of the joint's parts. This makes the articulation more firm as well as allows a fine joint calibration. In addition, we added two springs, which increase the contact force between the circular surfaces. Connecting the spring ends to the centre of curvature of the two profiles, results in a constant springlength twice the radius of the profile. In this case no torque 


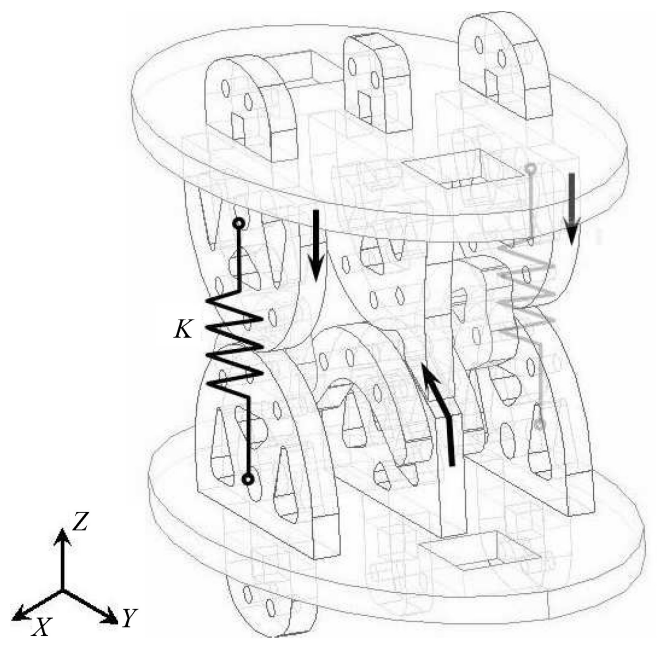

(a)

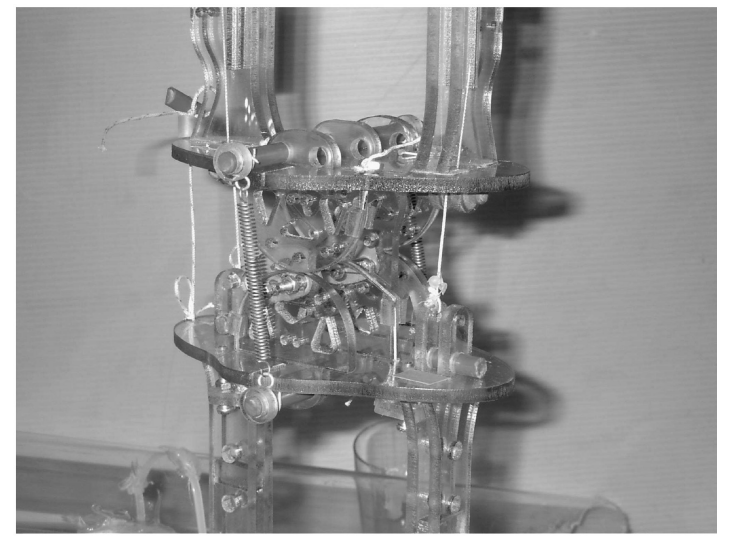

(b)

Figure 2. (a) The knee joint designed for our robot. The arrows show the way the tendons are wrapped. (b) The knee prototype

interferes with the joint rotation. Anyhow, it is possible to arrange the springs in a way that they force the joint to rotate to a particular equilibrium position.

In the knee we arranged the springs as shown in Figure 2 during rotation, the spring length has a maximum for $\theta=\bar{\theta}$ (see figure 3 ), where $\theta$ is the angle that the shank forms with the vertical. In the figure, $\gamma$ represents the angle between the shank axis and the force direction. When this angle is zero, the springs torque is zero too and $\theta$ equals $\bar{\theta}$. This permits to find an instable equilibrium position, as the spring is at its maximum extension. Attaching the spring forward, with $\psi<0$ (see figure), we can both help knee bending during leg swinging and knee stretching at the end of the step.

This kind of knee articulation has several advantages over a pin joint. The first is the energy efficiency, since we
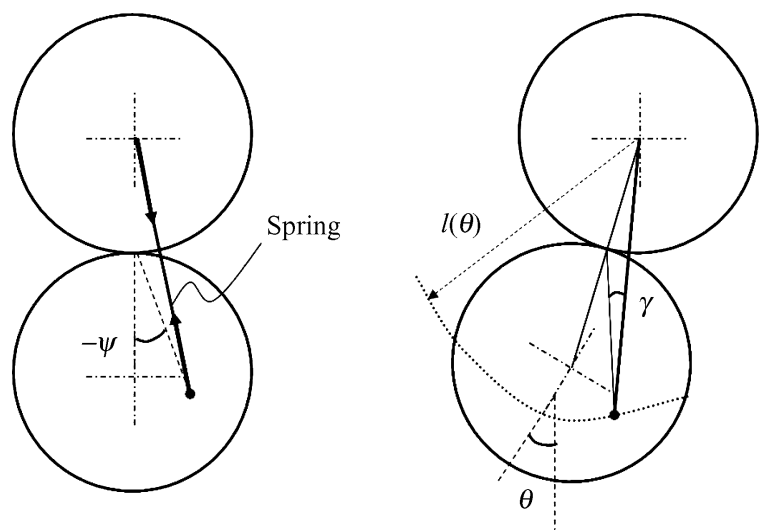

Figure 3. We can exploit the action of the springs to generate a position of instable equilibrium at $\theta=\bar{\theta}$, where $\bar{\theta}$ is the maximum spring length. In this way it is possible to favour both knee bending and knee stretching. reduce friction at the knee. Moreover, using elastic actuators or even a passive knee, the leg can be bent exploiting inertial forces due to hip actuation. Another aspect that strongly characterises this compliant joint is that the center of rotation (cr) is not fixed, as in a pin joint, but moves upward and backward during the rotation (Figure 4).

This motion increases the foot clearance necessary to swing the leg, and the shank active rotation can thus be reduced. The effect is both on energy consumption i.e. the knee could be passive in some robots - and on the gait stability, since the inertial load of knee-bending and knee-stretching is very important in the dynamics of walking. This is also the reason why the foot must be designed as light as possible, as described in the next subsection.

Regarding the radius of curvature of the two surfaces, we can look for an optimal design to maximise the foot

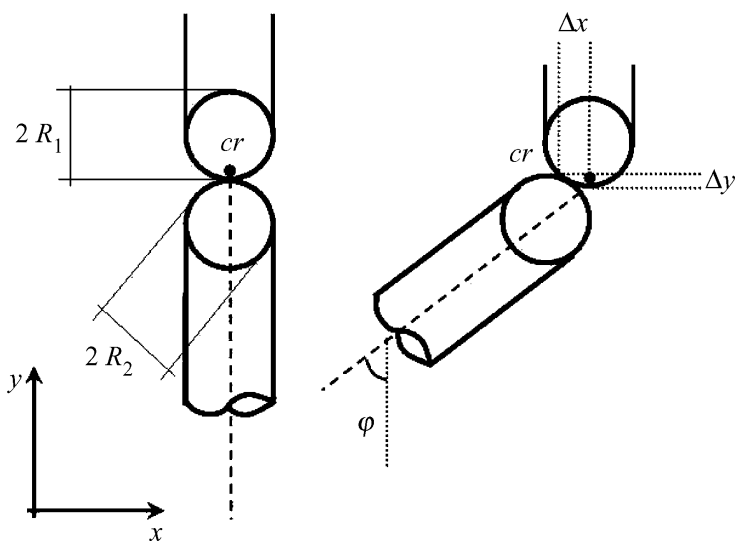

Figure 4. When the leg is bending, the centre of rotation moves upward and backward, according to the ratio between $R_{1}$ and $R_{2}$. 


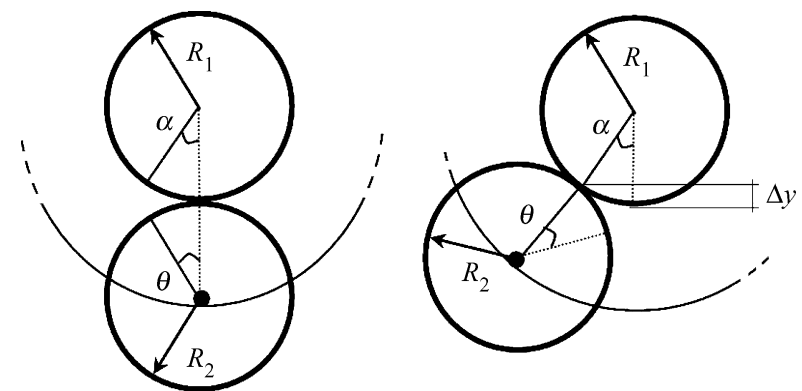

Figure 5. The upward movement can be expressed as a function of $R_{1}, R_{2}$ and the rotation $\theta$, due to the constrain of rolling without slipping.

clearance during the rotation. In case one contact surface (for example the upper one) has infinite or zero radius, the upward translation is null during the rotation. This means that there must be a finite rate value of the two radii that maximises the upward motion. Considering that the two surfaces are in contact without slipping, the upward motion $\Delta y$ can be expressed as (Figure 5)

$$
\begin{gathered}
\alpha R_{1}=\theta R_{2} \\
\Delta y=R_{1}(1-\cos (\alpha))=R_{1}\left(1-\cos \left(\frac{R_{2}}{R_{1}} \theta\right)\right) .
\end{gathered}
$$

If we consider $R_{1}$ fixed and we vary $R_{2}$, the maximum can be found for a fixed $\bar{\theta}$

$$
R_{2}=(\pi / \bar{\theta}) R_{1}
$$

$\bar{\theta}$ can be considered as the angle of the bent knee in the instant when the foot is closer to the ground.

According to this simple analysis, the radius $R_{2}$ should be longer than $R_{1}$, and the bigger the ratio between the two radii, the smaller will be the shank rotation, still having a flat surface rolling on the upper one.

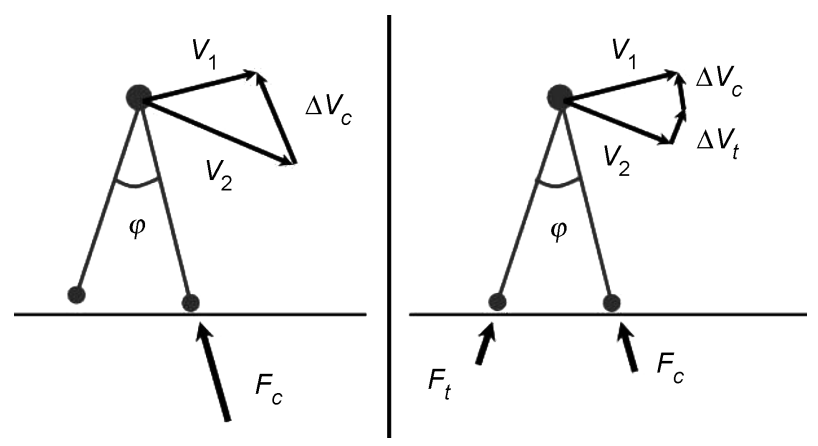

Figure 6. In the figure, $v_{1}$ and $v_{2}$ are the $\mathrm{cm}$ velocities respectively before and after heel strike, while $F_{c}$ and $F t$ are the ground reaction forces. With toe off (on the right) the $\mathrm{cm}$ vertical velocity is reduced, and the gait is smoother and more efficient.

\section{The role of the foot}

The foot is another challenging part for a biped robot to be anthropomorphic. Not only from a sensory point of view, but for the unique combination of mobility and lightness.

The foot inertia must be negligible with respect to the leg inertia. There are several evidences for this. One reason is the energy efficiency. During the swing-phase, the torque needed to move the leg forward is mainly due to inertial loads, that highly depend on the foot weight. In addition, these loads would act on the hip and the stance leg; their impact on the stability is more critical when the body weight/foot weight ratio is low. Low inertia is also fundamental for stability to keep the CM as high as possible. At the beginning of the stance phase, the biped robot can be considered as an inverted pendulum, both in the horizontal plane and in the frontal plane. A high CM increases the inertia of the pendulum with respect to the hinge. It is well known that this implies slower changes with respect to the initial position and thus a wider stability with respect to external disturbances (Sardain et al. 1999). As already pointed out about the knee, stability and energy efficiency are strictly related, since a more stable gait requires less motor action to counteract disturbances (Maloiy et al. 1986). Recently (Sellaouti et al. 2006) have compared a walking gait with and without a compliant foot and demonstrated a faster walking speed as a consequence of a toed foot.

Another aspect that characterises the human foot is its mobility and elasticity. Ker et al. found that the foot behaves like an elastic body, returning about $78 \%$ of the energy in its elastic recoil. While running, the arc of the foot stores and returns $17 \%$ of the energy the body loses and regains at each footfall, till the $35 \%$ of this energy is stored and returned by Achilles tendon (Ker et al. 1987).

Foot mobility has a big influence on the whole kinematics and dynamics of the walking motion, especially on the ankle. During the stance phase the contact point moves from the heel to the toe, and the foot is rotated before the toe off. The position of the contact force plays a very important role in determining the joint torques, thus the energy consumption. As in normal walking the ground reaction, due to the gravity, is much higher than inertial forces, in first approximation, we can consider only this force acting on the stance leg (Vaughan 1996). From this point of view, the bigger the lever between the joint and the contact force, the bigger would be the torque needed. In order to minimise energy consumption, while walking we naturally pose the leg joints close to the line of action of the contact force (Saunders et al. 1953; Alexander 1992). For this reason it is important to have a foot that adapts to the position of the ankle, and thus of the other joints, without losing grip.

This aspect is very relevant at toe off, when only a small region of the foot is in contact with the ground (Doneland et al. 2002; Kuo 1998). Figure 6 shows a simple biped model at heel-strike: the rear leg is in the stance phase, 
and the foreleg is about at foot fall. The energy loss at the impact depends on the vertical velocity of the $\mathrm{CM}$. The ideal situation is when the CM velocity is parallel to the ground, and the legs simulate the wheel (McGeer 1990). In normal walking, without toe off the motion of the $\mathrm{CM}$ is rotational along the contact point of the stance leg. This means that at footfall there is a component of CM vertical velocity that causes impact loss. Using toe off, this component can be significantly reduced, resulting in a more efficient and smooth gait. (Kuo 1998) figured out that providing all the energy necessary for walking by the toe off muscle instead of the hip reduces the energy cost by a factor of 4 .

\subsection{The design of the anthropomorphic foot}

Nowadays almost all the biped robots adopt a flatfoot, with relatively heavy dampers to smoother the heel-strike. A flatfoot imposes that the ankle position is fixed during the whole stance phase and, at toe off, the contact is reduced to the foot edge (Figure 7). This solution has the advantage of a simple design, and ensures a big base during the stance phase.

Another type of simple foot profile, adopted mainly on passive dynamic walkers, is the round foot. Its advantage is that the ankle joint is moved forward during the rotation, minimising the torque needed at toeoff. Its drawback is that the contact surface is reduced to a thin area, so this kind of foot is mainly adopted on two-dimensional bipeds.

Our goal was to develop a foot with the right tradeoff between mobility and stability, keeping the structure as light as possible at the same time. We adopted performing materials, polycarbonate and carbon fibre, and we designed the human-foot structure as a two-DoF device, as shown in Figure 8 . The foot has one passive degree of freedom that represents the heel, an arc and another passive DoF for the toe. In addition, we inserted an artificial Achilles tendon between the heel and the arc.

The articulations in the foot play an important role in gait kinematics and dynamics. As shown in Figure 8, at heelstrike and at toe off, the ankle position is not constrained in one fixed position. This gives the ankle an addition DoF,

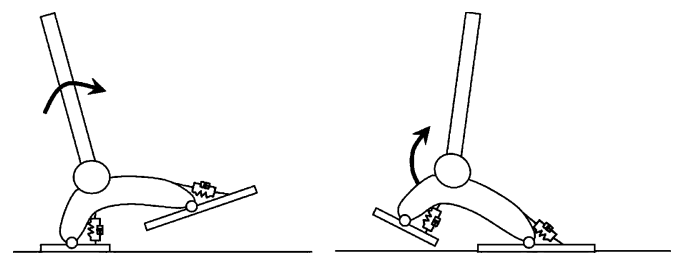

Figure 8. The foot developed to mimic the human one. It has two passive degrees of freedom, with a spring-damper system to smooth the heel strike.

which makes it possible to minimise energy consumption as well as to have a stable support for the robot. Generally speaking, during the stance phase the contact position moves from the heel to the toe. With our foot the $\mathrm{cr}$ follows the same motion. This means that the lever arm of the ground reaction force is already reduced with respect to a flat foot, where the ankle and the $c r$ are constrained in the same fixed point. Moreover, the foot keeps a firm base to lean even at toe off, when the ankle is moved forward and upward for knee bending. In this way the double support time - the time when both feet lean on the ground - can be increased, resulting in a more stable walk.

\section{Construction of the biped robot and development of the actuation system}

\subsection{The LARP prototype}

The biped LARP integrates the design of the knee and of the foot as illustrated earlier. LARP (Figure 9) (Scarfogliero et al. 2004) is $90 \mathrm{~cm}$ tall and weights less than $5 \mathrm{~kg}$. It is entirely made by pieces cut out from a polycarbonate sheet with laser cutting technology. Polycarbonate is a polymer that has a good strength-weight ratio, and can be widely deformed before breaking.

Figure 10 shows the organisation of the twelve active DoF. The hip joint, not discussed here in detail, has three orthogonal DoF. The design of LARP is studied to limit the room needed by the joints, also considering that the motors are not directly applied to them.
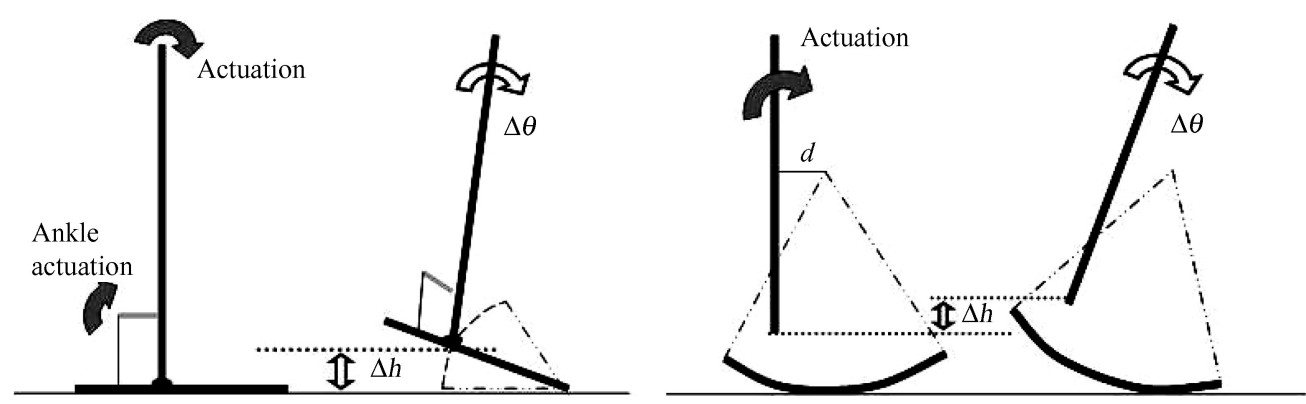

Figure 7. The flatfoot compared to a circular foot. 


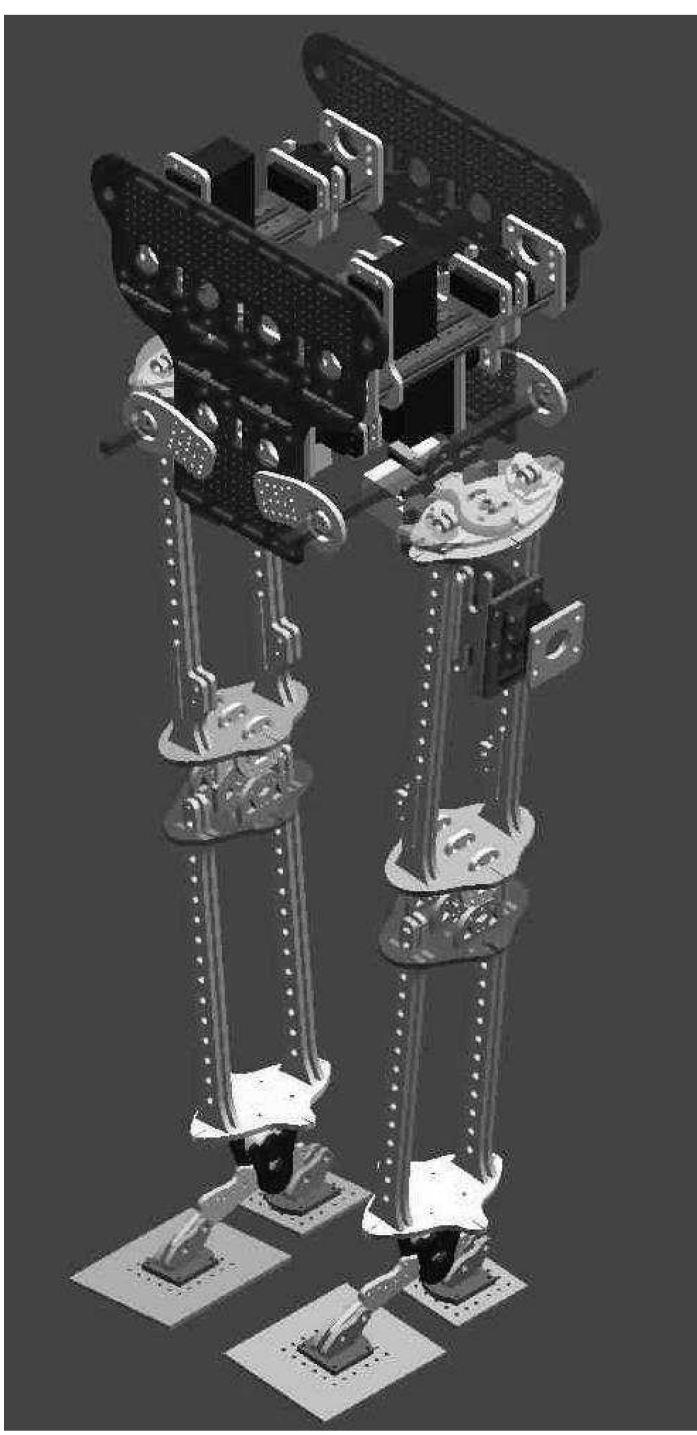

(a)

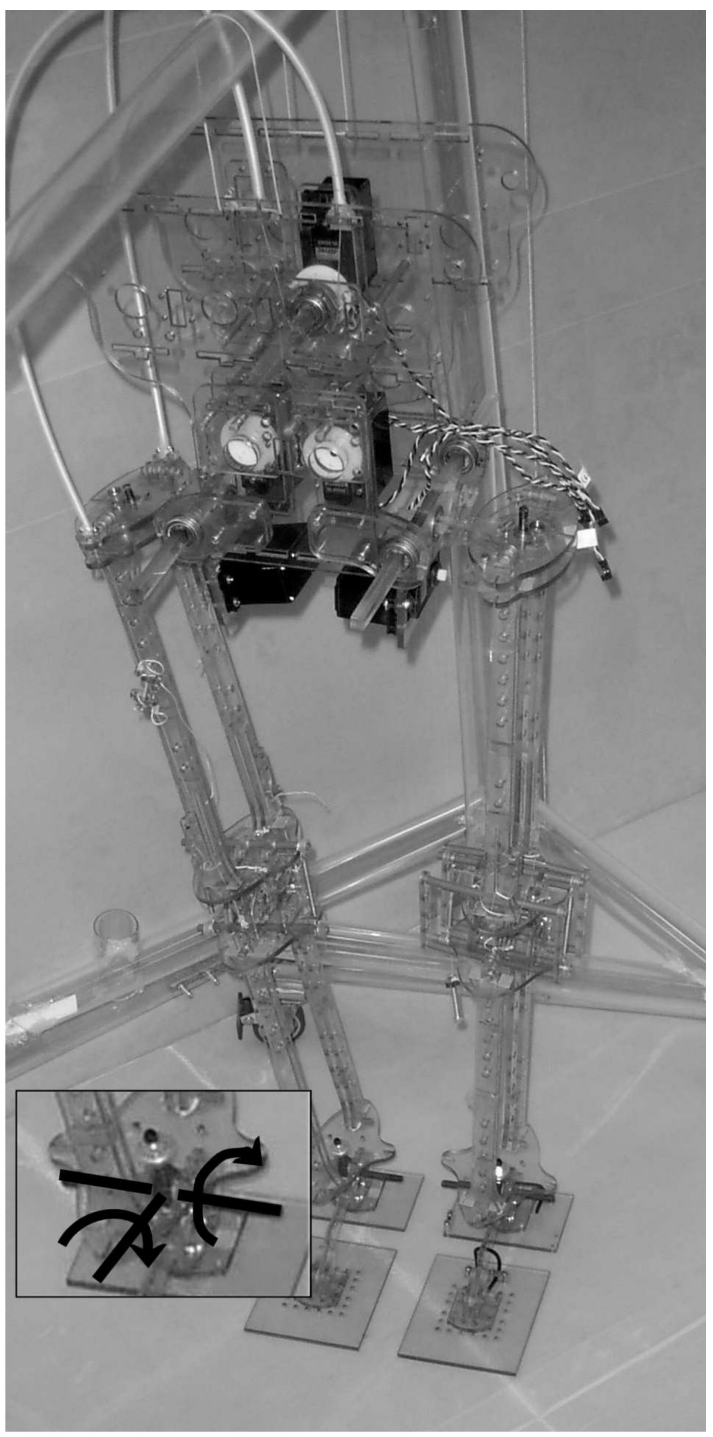

(b)

Figure 9. (a) The 3D model of the robot. (b) The prototype. In the low part of the picture is a detail of the two rotational joints located in the ankle.

The pelvis can host twelve servo motors connected by tendons to each joint of the robot. As some motors are included in the upper part of the thigh, there is also spare room for the actuation of an upper part of the robot.

All the servo motors are equipped with a spring and a damper to control the joint stiffness, as illustrated in the next subsection.

\subsection{The spring-damper actuator of $L A R P$}

Each actuator is composed of a servo motor with $2.4 \mathrm{Nm}$ torque, a torsional spring, and a torsional pneumatic damper. The resulting actuator has a good shock tolerance, fundamental in walking, as impacts occur at every step. In addition, we can exploit the natural dynamic of the link storing energy in the spring. Similar actuators, with a DC motor and a spring, have been successfully used in biped robotics (pratt and Williamson 1995; Yamaguchi and Takanishi 1997).

In this actuator the joint stiffness is not infinite, as in servo motors, and can be changed in real time despite the constant stiffness of the spring. This requires a good choice of spring-damper characteristics and a proper control algorithm.

Let $k_{g}$ be the joint stiffness (Equation 4)

$$
k_{g}=\frac{T_{e}}{\varepsilon},
$$




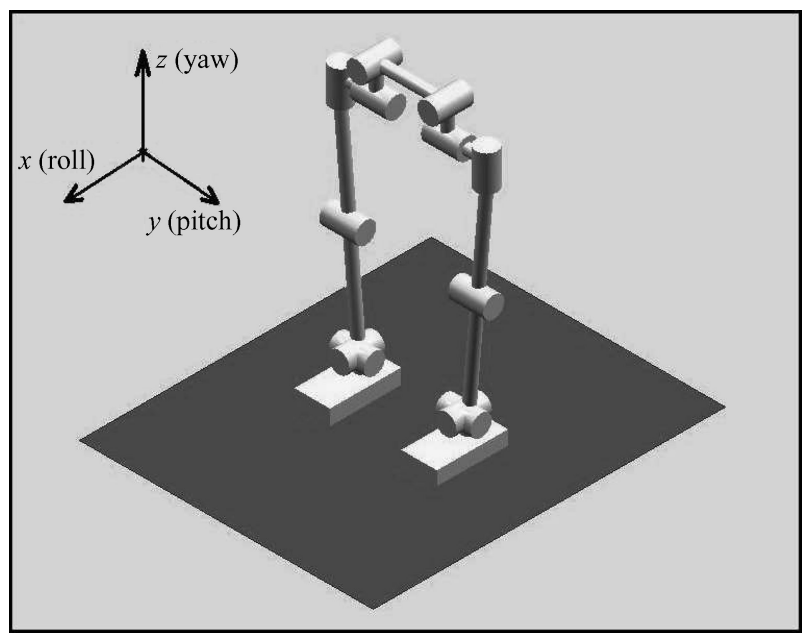

Figure 10. The organisation of the twelve degrees of freedom (DoF) in LARP.

where $T_{e}$ is the external load and $\varepsilon$ is the position error. The initial stiffness of the joint is fixed by the spring constant, because the motor needs some time to tension the spring and counteract the external torque. In this condition the damper in parallel with the spring permits to avoid high initial errors due to rapidly varying loads.

The damping factor $d$ can be set as constant and its critical value setting the constant $(\xi=1)$; in equation $5, w_{n}$ is the natural frequency of oscillation, and $I$ is the inertia moment of the joint.

$$
w_{n}=\sqrt{k_{g} / I} d=2 \xi w_{n} I
$$

or can be changed during motion operating by a proportional electro-valve that regulates the air flux between the two chambers of the damper, in order to save motor torque and make the system faster.

\subsection{The control algorithm for a constant damping factor}

The spring-damper actuator can be used in a torque control loop; the high-level controller assigns the torque to be delivered and, measuring the spring deflection, the low-level regulator makes the actuator perform the task.

A way to assign joint torques is the Virtual Model Control (Pratt et al. 2001), where the controller sets the actuator torques after the simulation on a virtual mechanical component. In other approaches (Kwek et al. 2003) the joint torque is computed from the dynamic model of the robot. The biped robot can be formalised as a multi-input-multioutput (MIMO) non-linear system, that sometimes presents time variant dynamical behavior. In these conditions a classical Proportional Integral Derivative (PID) controller is not suitable. Moreover, if we apply a simple position controller we could not control the joint stiffness.

We designed two algorithms to control the joint stiffness and position, as illustrated in the following.

(1) The basic controller: The basic control algorithm is close to the classical model of the Equilibrium Point Hypothesis. It takes the reference position $\bar{\varphi}$ and the joint stiffness $k_{g}$ as inputs, and outputs the motor position $\alpha_{0}$. The only state information needed is the actual joint position, that must be measured and feed-backed to the regulator. The difference between the actual position and the motor position is covered by the spring deflection.

The control law is expressed by Equation (6)

$$
\alpha_{0}=\frac{k_{g}}{k}(\bar{\varphi}-\varphi)+\varphi
$$

where $k$ is the spring stiffness, $\varphi$ and $\bar{\varphi}$ the actual and desired angular positions. The result is that a virtual spring with $k_{g}$ stiffness is acting between the reference angle and the actual position. If $k_{g}=k$, we get $\alpha_{0}=\bar{\varphi}$, as the spring and joint stiffness coincide.

If $k_{g}<k$ the motor rotation is lower than the reference, as the spring stiffness is too high. If $k_{g}>k$ the motor has to rotate more to generate higher torques. Thus, $k_{g}>k$ attenuates the effects of a motor position error, while $k_{g}<k$ is suited when the motor limits in the speed. To avoid high initial acceleration $\bar{\varphi}$ can be defined with a second order function.

The error in reaching a position is small for high stiffness. If stiffness is small, instead, one may expect relevant differences between the reference and actual trajectories.

The static error $\epsilon$ depends on the external load $\left(T_{e}\right)$, as in Equation (7)

$$
\epsilon=\frac{T_{e}}{k_{g}}
$$

Equation (7) represents also a way to find the joint stiffness, deciding the maximum error tolerance and estimating the external maximum load. Note that $k_{g}$ can be changed in real time.

We illustrate the control of a simple 1-DoF pendulum. The system parameters are

$$
\begin{aligned}
m & =1.2 \mathrm{~kg} ; l=0.3 \mathrm{~m} ; I_{g}=7.35 \cdot 10^{-2} \mathrm{Kg} \mathrm{m}^{2} ; \\
k & =6 \mathrm{Nm} / \mathrm{rad} ; \mathrm{kg}=10 \mathrm{Nm} / \mathrm{rad} .
\end{aligned}
$$

where $l / 2$ is the distance between the $\mathrm{CM}$ and the joint axis. We defined the reference trajectory with a step function filtered by a second order filter with a time constant $T$. The damping factor was set to keep the system at the critical damping, as in Equation (5).

Figure 11.(a) shows the joint angle and the motor position for the movement from 0 to $0.3 \mathrm{rad}$ at $0.1 \mathrm{~S}$, and from 


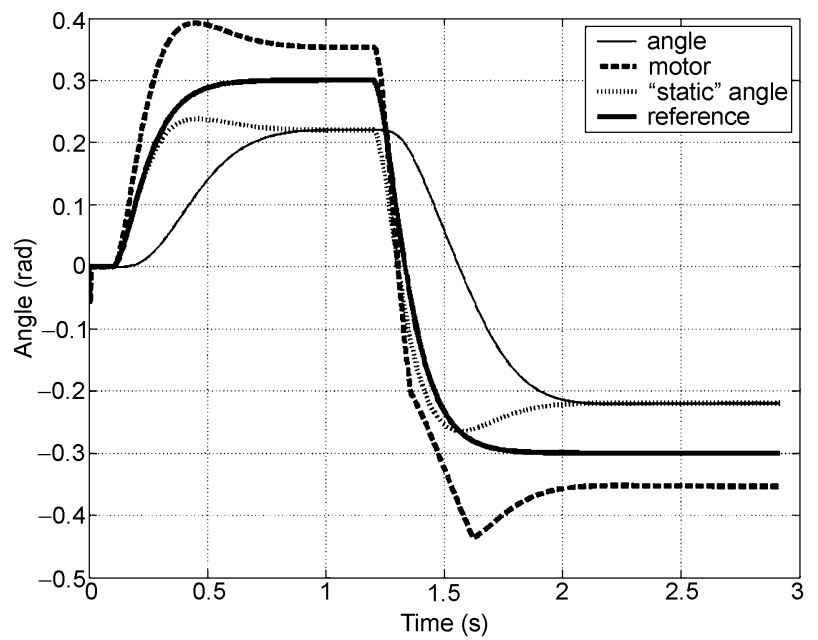

(a)

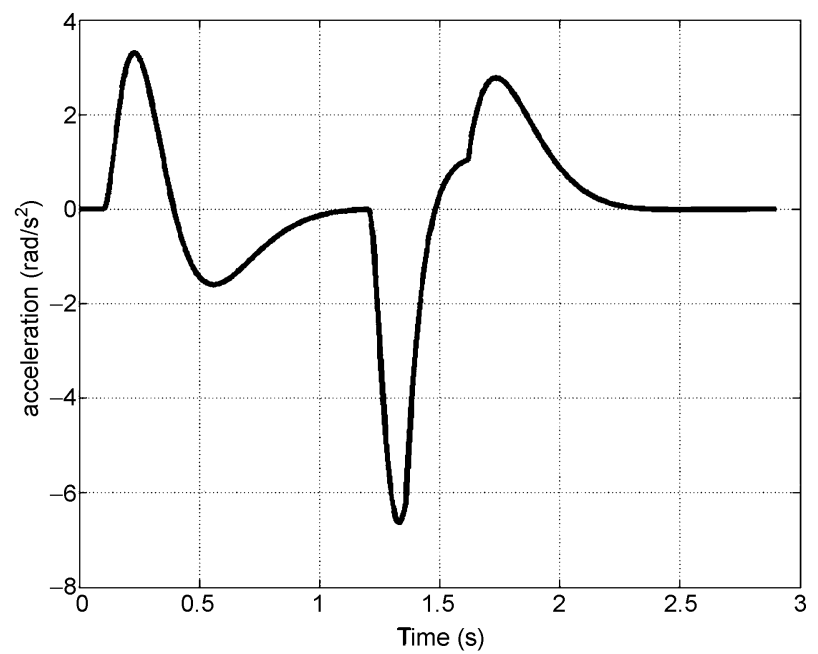

(b)

Figure 11. (a) The link rotation and the motor position for the movements from 0 to $0.3 \mathrm{rad}$ and from 0.3 to $-0.3 \mathrm{rad}$. The actual angle approaches the reference according to stiffness and external load ('static' angle). (b) The acceleration pattern with two peaks, characteristic of damped systems. The change at about $t=1.4 \mathrm{~s}$ is due to the limit on servo maximum torque.

0.3 to $-0.3 \mathrm{rad}$ at $1.2 \mathrm{~S}$, with a constant time $T=0.08 \mathrm{~s}$, and only considering gravity. With 'static angle' we denote the position that the joint would have if the link inertia was zero and no damper present. The chosen stiffness is low and the error, due to gravity, is about $0.1 \mathrm{rad}$. The motor position is opposite to the angle with respect to the reference, since the spring stiffness is chosen lower than the joint stiffness; the motor has to rotate more but the system is less sensitive to motor position errors. At about $1.4 \mathrm{~s}$, the motor rotation changes velocity due to maximum torque limit of the servo.

The resulting acceleration, in Figure 11.(b) shows only two peaks, acceleration and deceleration with no oscillation. This pattern, typical of damped systems, is useful to exploit the natural dynamics of multi-link systems. For in- stance, when starting a step, the acceleration of the thigh can be used to bend the knee, as in passive dynamic walkers (McGeer 1990) (Collins et al. 2001), or, before footfall, the deceleration of the swing motion can be exploited to straighten the leg, as in passive lower-limb prosthesis.

Using this simple control law we do not need to solve any inverse dynamic problem, but just decide the joint stiffness - using for example Equation (7) - and define the reference pattern.

The case is different when we want to follow a given reference trajectory controlling the motor torque. To this end we developed the algorithm in the following.

(2) Force estimation through acceleration feedback. To consider acceleration constraints we developed a sort of impedance control, the algorithm tracks the delivered torques and relates them to the resulting acceleration. In this way we create a simple dynamic model of a multi-body system without solving any inverse dynamic problem. The model can also get a good estimate of the external load acting on the joint, both due to gravity and to interactions with another link.

We use in the control loop the equations

$$
T_{e}^{i-1}=-k \cdot\left(\alpha_{0}^{i-1}-\varphi^{i-1}\right)+I \cdot \ddot{\varphi}^{i-1}+d \cdot \dot{\varphi}^{i-1},
$$

where $d$ is the damping factor $\alpha_{0}, I$ is the inertia and $k$ the elastic constant. We assume a constant external load between the instants $i-1$ and $i$

$$
T_{e}^{i-1}=T_{e}^{i} .
$$

Given $k, d, I$, the position of the motor $\alpha_{0}$ and the estimation of $T_{e}$, the acceleration is computed

$$
A^{i}=\frac{k \cdot\left(\alpha_{0}^{i}-\varphi^{i}\right)+T_{e}^{i-1}-d \cdot \dot{\varphi}^{i}}{I} .
$$

This is a kind of impedance control if the acceleration (system output) in the next step is different from the foreseen one, given the calculated $\alpha_{0}$ (system input), it infers that a different load is acting (system model has changed) and the motor position $\alpha_{0}$ is corrected.

The simulations on a single joint, defined as before, are shown in Figure 12 with and without motor torque limitation, considering only gravitation. As illustrated in Figure 12(c) the characteristic is similar to the human electro-myographic activity, composed by their phases acceleration-pause-deceleration (Kiriazov 1991), (Gottlieb et al. 1989), and suitable for exploiting the natural dynamic of the links, i.e. in leg swinging. From Figure 12(e) and (f) we can also notice that the system performs a pretty good estimation of the external load acting on the link.

The controller can also monitor the acceleration along the path; if we impose a joint stiffness too high or if the 


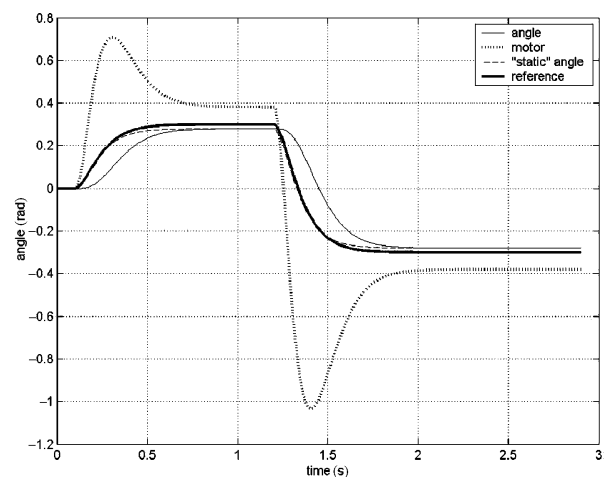

(a)

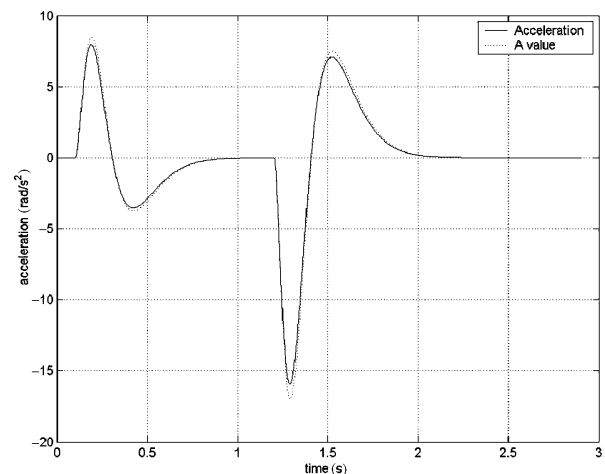

(c)

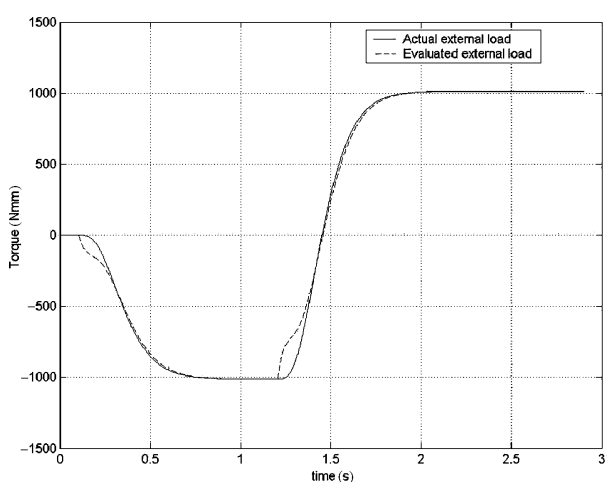

(e)

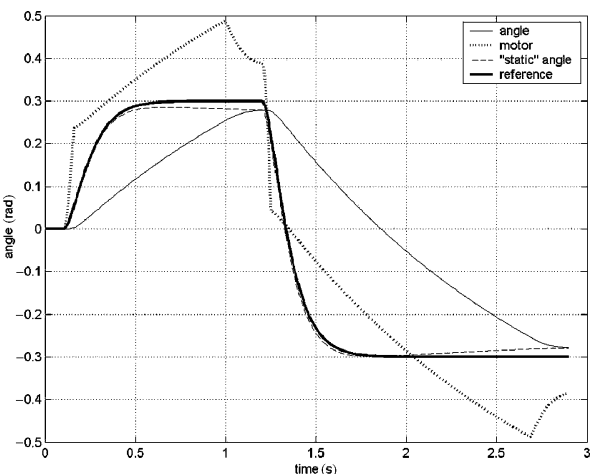

(b)

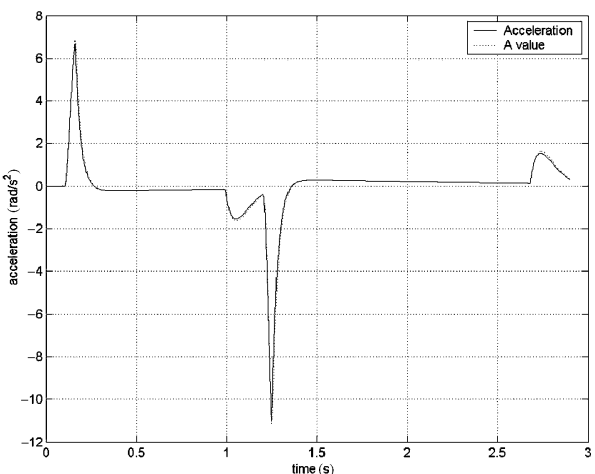

(d)

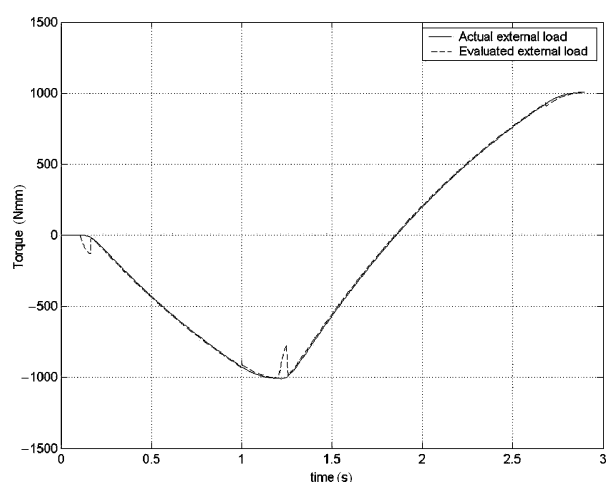

(f)

Figure 12. (a),(c),(e) show the angles, the acceleration and its evaluation, $T_{e}$ and its estimation whit no motor torque limitation. (b),(d),(f) show the same quantities when torque limitation is considered.

reference angle changes too quickly, the controller decreases the joint stiffness to reduce the acceleration. It uses the calculated acceleration with the imposed stiffness for the next iteration (Equation 10); if the acceleration $A^{i}$ is too high, the low-level controller modifies $k_{g}$.

The joint stiffness can be set from Equation (7) or with a trial-and-error procedure. The only information the controller needs is system inertia; in multi-link systems it can be approximated with a constant average value computed on all the links, or can be calculated during the motion.
Regarding the damping factor, Equation 5 can be rewritten as

$$
d=2 \xi \cdot \sqrt{k_{g} I}
$$

and shows that the damping factor is proportional to the square root of inertia errors; while a too high inertia makes the system over-damped, an underestimation can let the system oscillate. Anyway the error in the inertia must be as high as $50 \%$ to see noticeable effects on the damping. In the external torque estimation (Figure 14), we can notice 

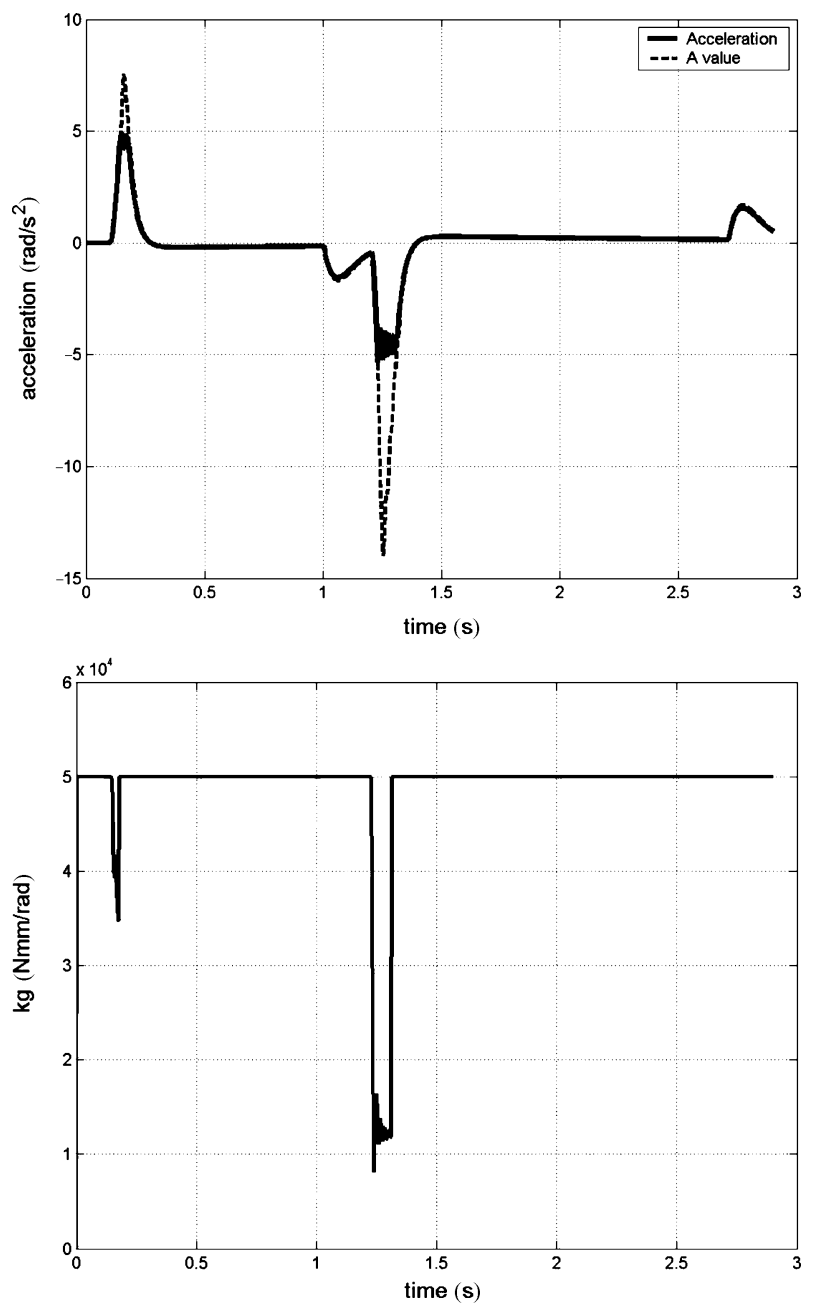

Figure 13. The algorithm can limit the acceleration acting on the joint stiffness without compromising the final positioning.

the effect of wrong inertia value. If the real inertia is higher, the controller acts as an additional external load.

\subsection{Results in simulation of some behaviors of the biped}

(1) Maintaining the equilibrium: The spring-reactive control has been coded into a computer simulator of the 3D model of the robot; this model is shown in the left side of Figure 9. In all the simulations the floor was considered as a flat surface with a defined and finite stiffness; the two flat parts of the feet were represented by four spheres each. This was done in order to reduce the number of contact points during the stance and support phases, and speed up the simulations. Kinematic and dynamic parameters, and material properties were properly taken into account. The simulations were done integrating the Adams ${ }^{\circledR}$ software capabilities with the Matlab-Simulink ${ }^{\circledR}$ environment, thus considering all the dynamical features of the real robot and, at the same time, using a powerful framework to test our control strategies.
In Figure 15 we test how the robot preserves equilibrium despite external disturbances; we applied noise forces and torques having directions located in the robot's horizontal plane. To run this test we reduced the system complexity; since for this first experiment $6 \mathrm{DoF}$ are enough, we only actuate two DoF in the ankle (pitch and roll) and one in the hip (yaw) for each leg.

The joint stiffness is set according to Equation (7), where $\varepsilon$ is the maximum error and $T_{\text {ext }}$ is the corresponding gravitational load. Given the inertia $I$, we evaluate the needed total damping factor $d$. As in the feet two dampers are in parallel, we split the inertia so the sum of the two dampers equal the total damping needed. For the hip we proceed in the same way, neglecting the leg beneath the joint.

The results are shown in Figure 16 as the disturbance is applied, a position error appears. The dotted line shows the motor rotation that counteracts the disturbance and brings the joint back to the reference. In this way the robot is able to 'react' to external loads, accepting a positioning error in order to preserve its balance.

(2) Estimation of energy consumption: Here we present some preliminary energetic considerations obtained using the direct/inverse kinematic model of our biped. The walking surface is assumed flat and free of obstacles. Using the inverse kinematic solution we can set a reference trajectory for the foot and pelvis and calculate the joints positions.

The main idea is to set up a fast simulation environment that allow us to compare different kind of gaits from the kinematic and energy point of view. In these tests we worked in a static condition, therefore a further analysis that uses the more accurate dynamic model is required. This will allow us to evidence the real advantages of the complex mechanical architecture we chose for our robot.

Each gait we considered is characterised by the step length and height, the minimum height of the pelvis during motion, and the maximum lateral movement admissible (oscillations in frontal plane). Assume that during the motion the robot moves only between stable configurations, i.e. the projection of its $\mathrm{CM}$ falls inside the convex area that covers the contact surface of the two feet. The static stability is guaranteed by an algorithm that adjusts the pelvis position when the stability condition is not verified (see for details (Scarfogliero et al. 2004)).

In order to have a first estimation of the energy required for the motion we made the following assumptions and approximations

- Each link is modelled by a mass located in its barycenter.

- The $\mathrm{CM}$ for the entire robot is a weighted average of the links' CM.

- The robot moves slowly, therefore inertia forces are neglected. 


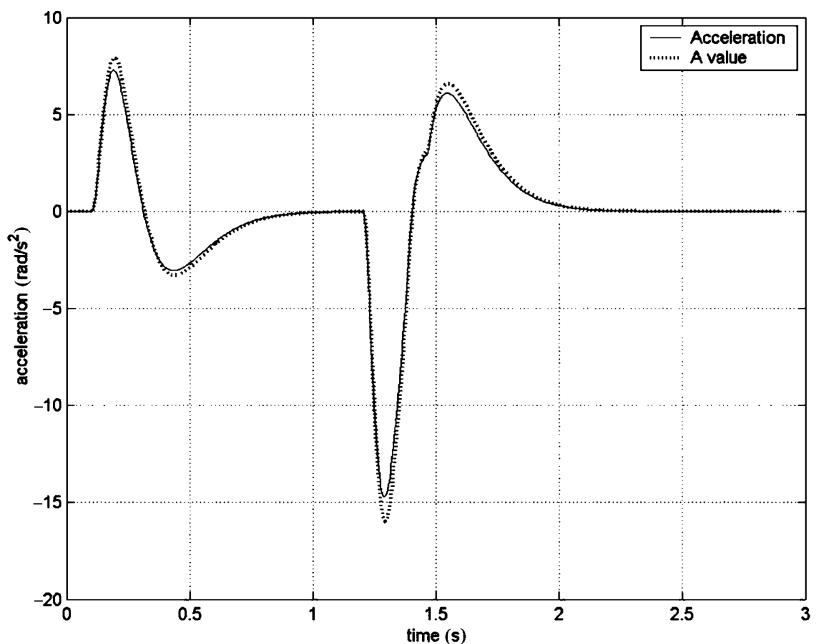

(a)

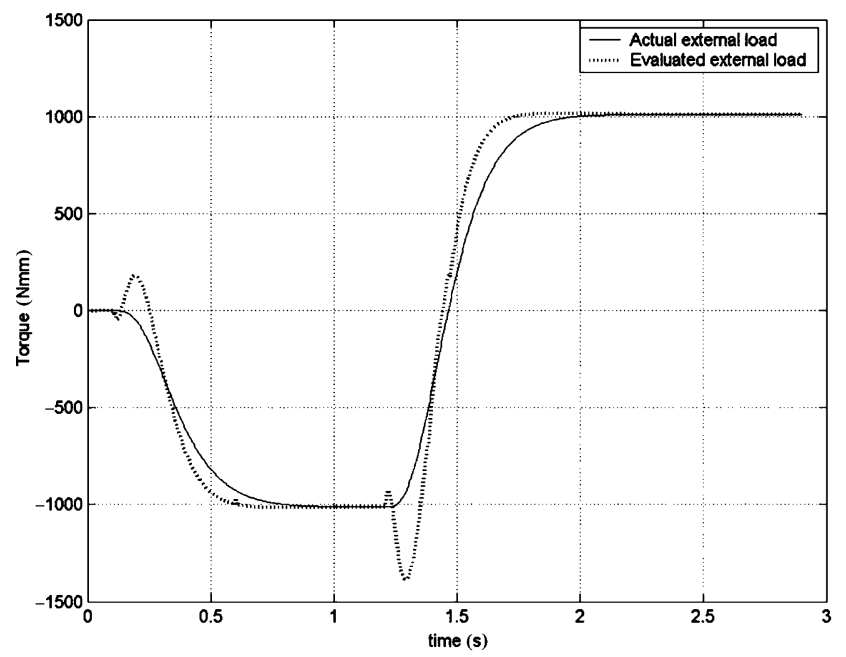

(c)

\section{Overestimated}

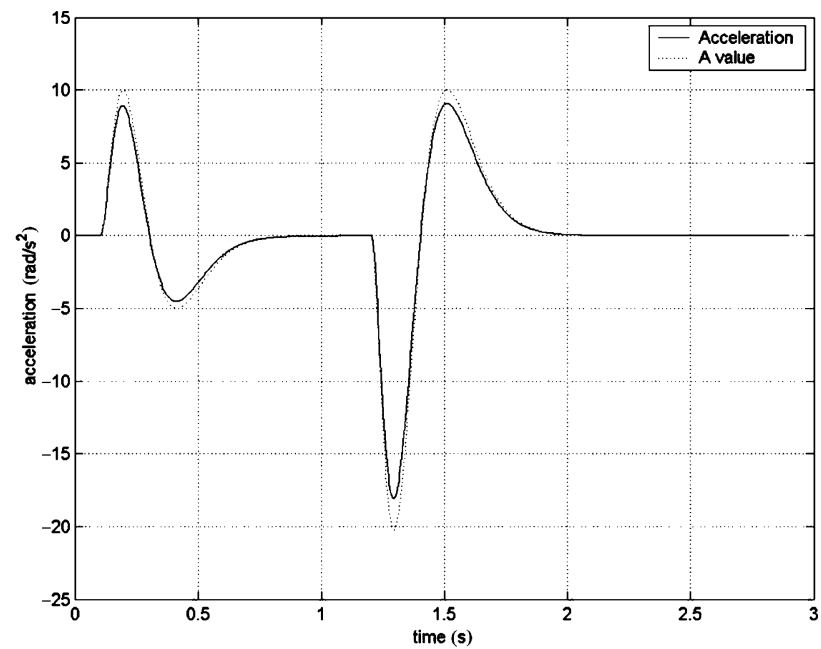

(b)

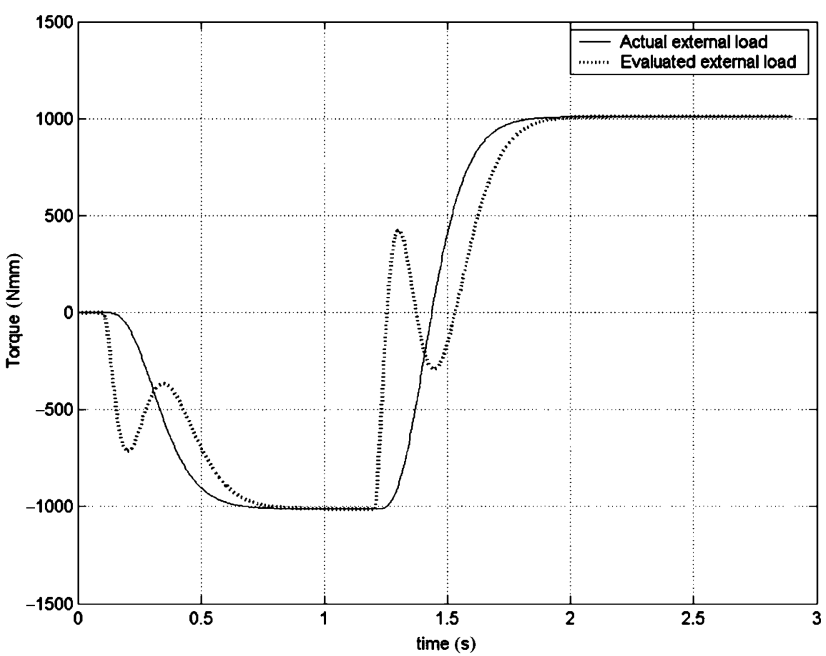

(d)

\section{Underestimated Inertia}

Figure 14. An error of $30 \%$ in inertia value does not compromise the positioning. If the inertia is underestimated, for example when accelerating, the algorithm interprets the too small acceleration as an external load. When is overestimated, a virtual additional positive torque is considered.

- We do not consider friction forces in the joints.

- We consider that kinetic energy during the falling phase (the food lifted approaches the ground) is completely lost.

The energy to lift each link was calculated with Equation 12 , where $m_{i}$ is the mass of the link-i, $g$ the gravity constant and $\Delta h_{i}$ the excursion along the $z$-axis for the $\mathrm{CM}$ of link-i.

$$
W_{i}=m_{i} g \Delta h_{i}
$$

The settings in the simulation of Figure 17 are step length $(0.5 \mathrm{~m})$, minimum height for the pelvis $(0.68 \mathrm{~m})$, and maximum lateral excursion $(0.09 \mathrm{~m})$.
The first two graphs on the left of figure 17 show the $Z$ coordinates for the pelvis and the foot during motion (in blue the reference trajectory, in green the real). In the third graph on the left of figure 17 we see the total energy consumed, about 2 joules. We note a significant deviation from the reference as a result of the stability algorithm. On the right side we see a stick model for the robot in the lateral plane.

In the second simulation we changed the minimum height for the pelvis at $0.55 \mathrm{~m}$. As illustrated in Figure 18 now the reference trajectory for the foot is well followed, but the energy spent increased up to 6 joules, since the links of the robot have a greater excursion along the $z$ axis.

These results confirm that lowering the barycentre to control the robot stability in static conditions results not 

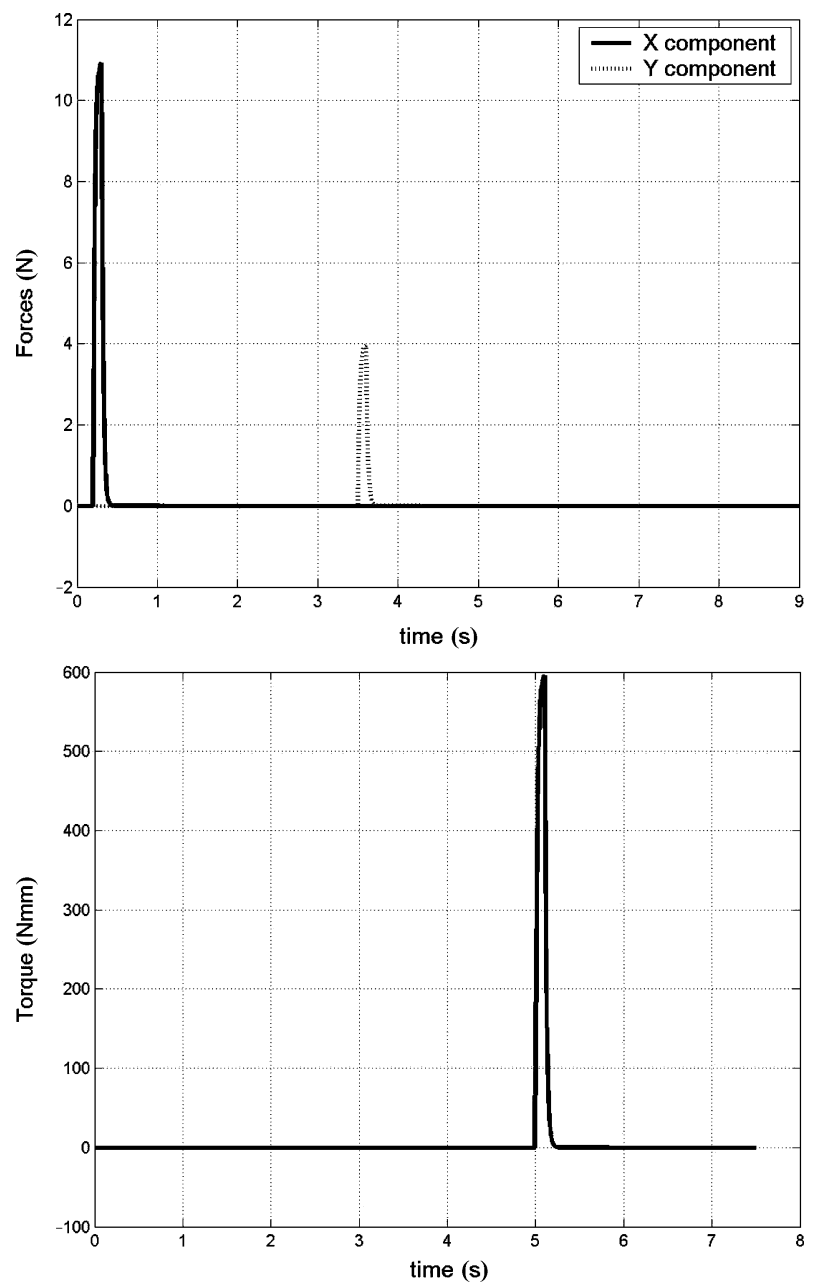

Figure 15. The external disturbances applied to the robot, forces and torque.

only in slow movements but also in high energy consumption. Human walking, on the contrary, is dynamical and the pelvis is maintained as high and as fixed as possible. The theory is in accordance with our results. Further analysis is needed to define the energy consumptions according to some global measure.

\section{Discussion and Conclusions}

Today several humanoid robots are able to walk and perform human-like movements. However the structure of such robots significantly differs from humans. This causes the robots to be energetically inefficient, as they are unable to exploit the natural dynamics of the links, and very poorly adaptable to unstructured terrain.

Studying the human knee and foot we found several advantages in adopting human-oriented design for these parts. In particular, a compliant knee was developed, having two circular contact surfaces and five tendons. This articulation demonstrates to be highly efficient and permits to increase
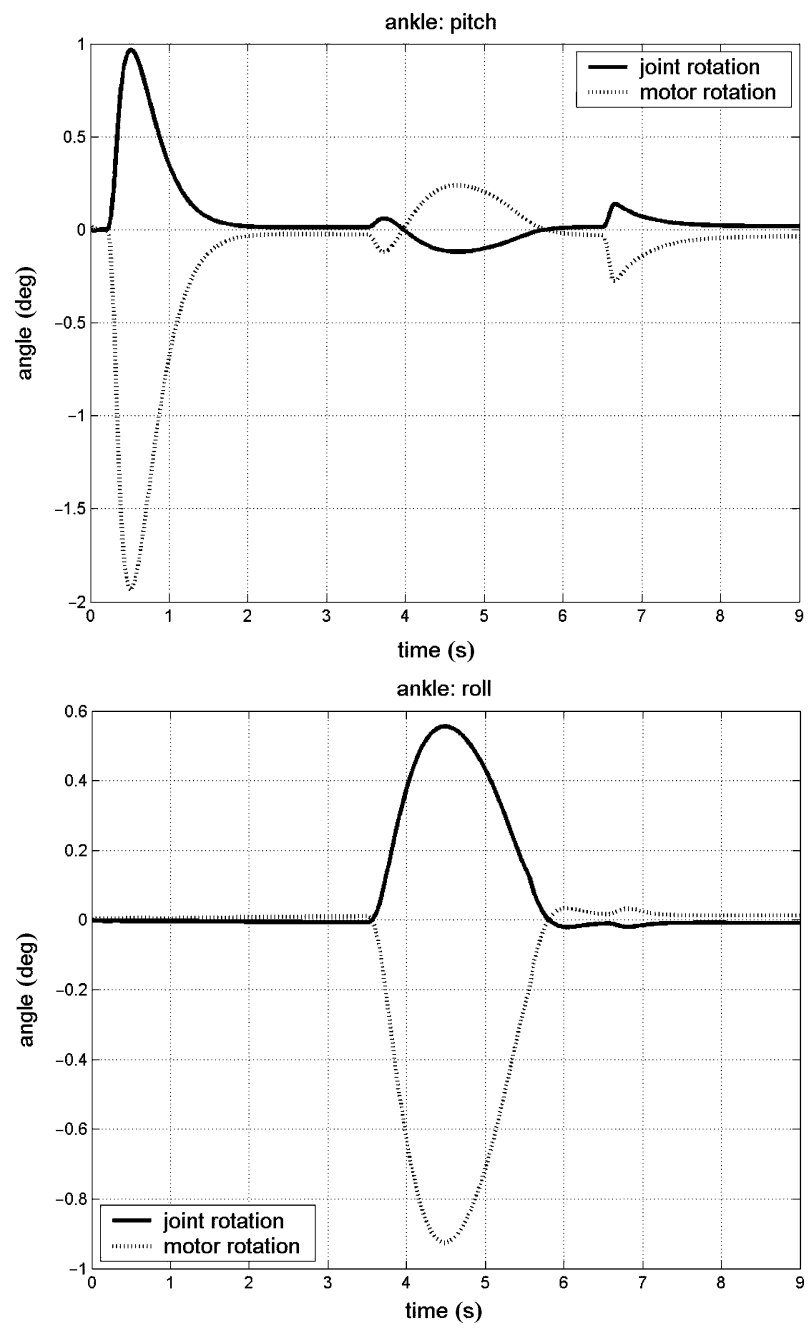

Figure 16. The angular position in the two degrees of freedom (DoF) of the ankle: the disturbances are absorbed and the robot returns in its initial position.

the foot clearance during the swing phase. Regarding the foot, two passive joints were introduced to mimic the high mobility of the human foot. An important limit for the foot structure was its weight, to be as light as possible. To ensure stability both at heel strike and toe off we used two planar surfaces connected to the arc of the foot by two passive DoF.

The movement control of the biped robot was performed using elastic actuators and a controller based on the Equilibrium Point Hypothesis (Scarfogliero et al. 2005). Regarding the actuation system, we designed a device equipped with a torsional spring and a damper. This allows to have a good shock tolerance and to estimate the external load measuring the spring deflection. Also, a method was developed to preserve the possibility of position control even with variable joint stiffness. This aspect is fundamental in biped robotics, not only to exploit the natural dynamics of the legs, but also to deal with impacts occurring at every step. In this context we implemented a sort of impedance control that let the 

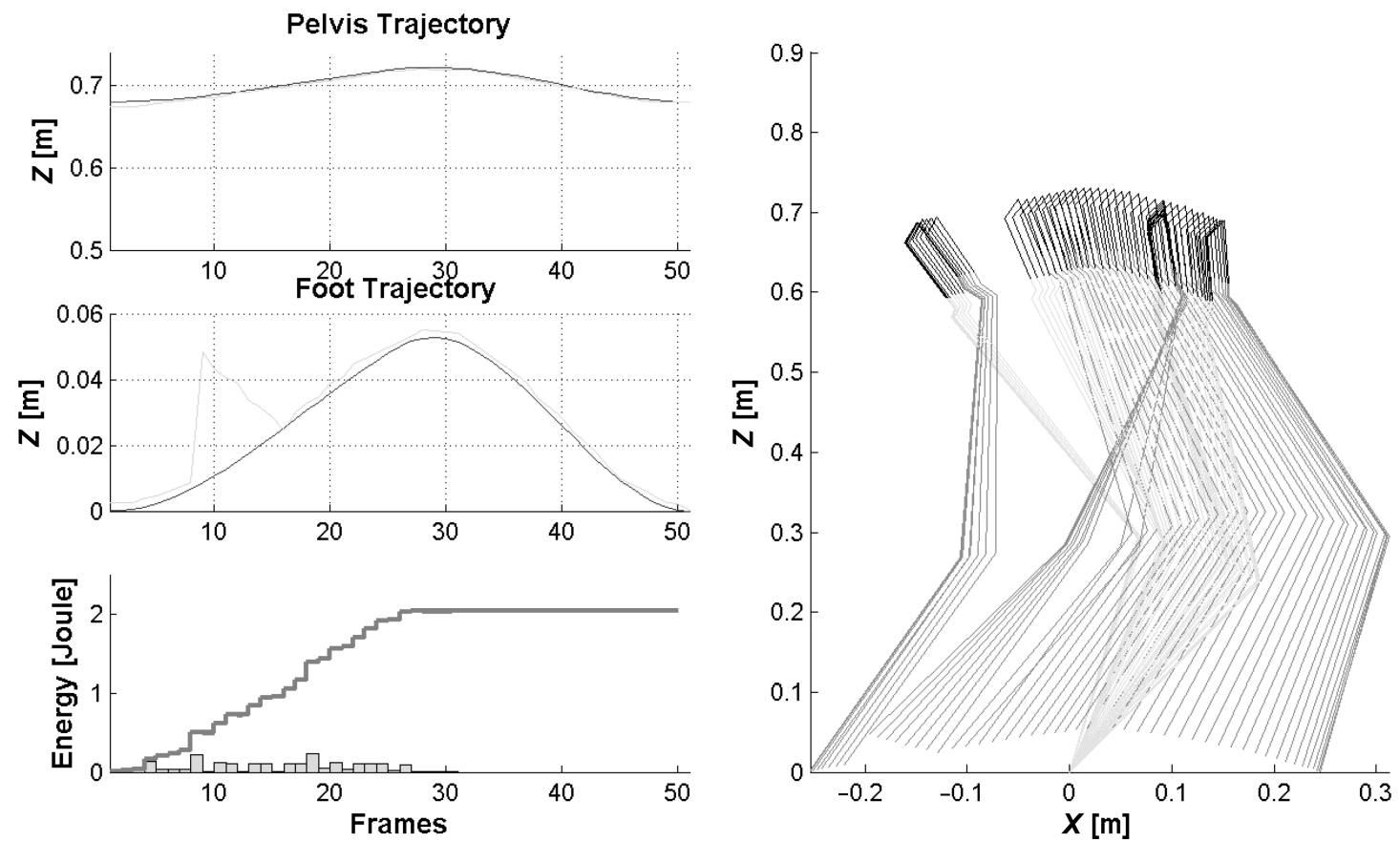

Figure 17. Energy consumption with minimum pelvis height $=0.68 \mathrm{~m}$.

low-level regulator modify the assigned stiffness. Doing so, for example, we can avoid high accelerations in real time and obtain a good estimation of the external load. In addition, the regulator demonstrated to be particularly robust in respect to system uncertainties, such as inertia values.
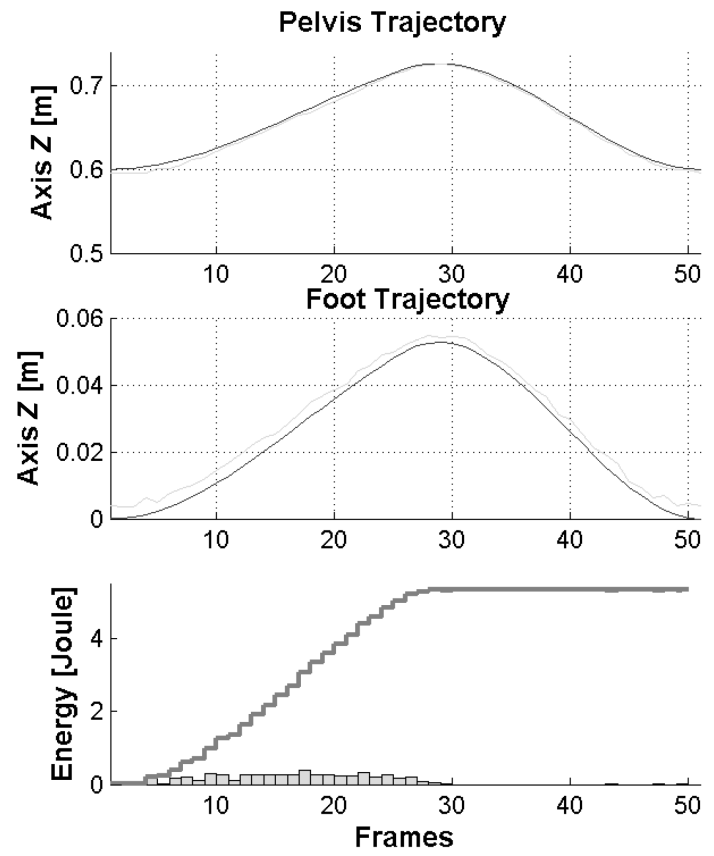

Comparing the resulting control law with existing models, we found several similarities with the Equilibrium Point Hypothesis. Deeper researches can be made in this sense, using the system we developed as a model and studying the influence of changes in the control parameters. Further

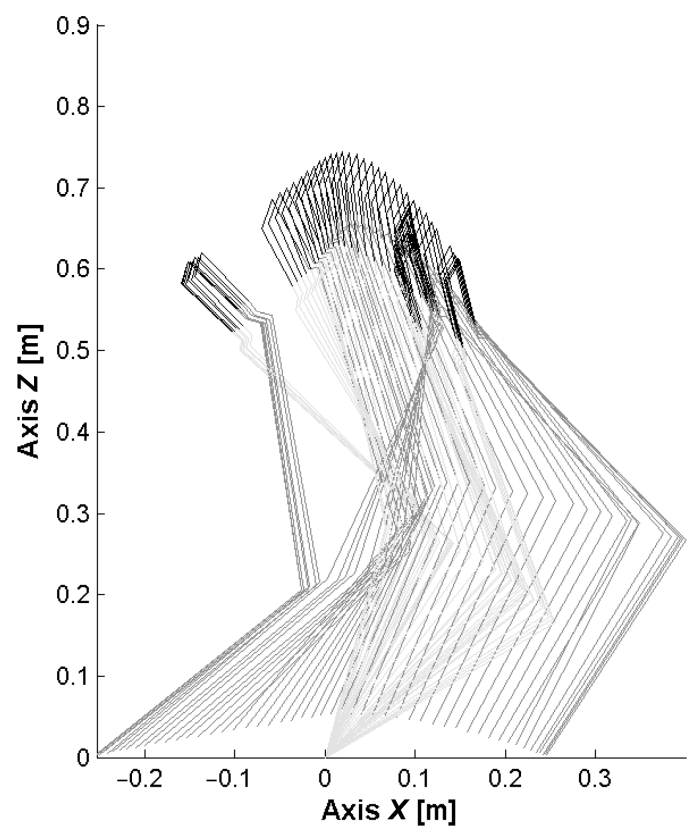

Figure 18. Energy consumption with minimum pelvis height $=0.60 \mathrm{~m}$. 
work can investigate the damper influence on the motion. In our simulations, to avoid oscillations along the assigned angle, the damping factor was fixed at the critical value. Thus, an alternative way is to choose the damping factor as an additional input parameter, to be controlled during the motion. According to the external load, the regulator could assign the damping needed to avoid oscillations and perform the right movement. It is also required to test our control strategy on the real joint and compare results with other control techniques.

We conducted a first gait analysis of the biped using a simplified static model and the framework we developed can be used to guide further more detailed simulations in dynamical condition.

Further work has to be done for the complete design of a human-like robot, starting from a new design of hip and ankle articulation. In this case we should investigate the role of the third DoF in the human ankle (torsion of the foot along the leg axis), which is omitted in most of the modern humanoid robots.

\section{References}

Alexander R, 1992. The Human Machine. New York: Columbia University Press.

Asatryan D, Feldman A. 1965. "Functional tuning of the nervous system with control of movement or maintenance of a steady posture - i mechanographic analysis of the work of the joint or execution of a postural task." Biofizika. 10:837-846.

Collins S, Wisse M, Ruina A. 2001. "A three dimensional passivedynamic walking robot with two legs and knees." Int J Robot Res. 20(7):607-615.

Doneland J, Kram R, Kuo D. 2002. "Simultaneus positive and negative external mechanical work in human walking." $\mathrm{J}$ Biomech. 35:117-124.

Fallis G. 1888. "Walking toy.” U.S. Patent No.376588.

Feldman A. 1966a. "Functional tuning of the nervous system with control of movement or maintenance of a steady posture ii controllable parameters of the muscle." Biofizika. 11:498508 .

-1966b. "Functional tuning of the nervous system with control of movement or maintenance of a steady posture - iii mechanographic analysis of the work of the joint or execution of a postural task." Biofizika. 11:667-675.

Garcia M, Chatterje A, Ruina A, Coleman M. 1998. "The simplest walking model: Stability, complexity and scaling." ASME J Biomech Eng. 120:281-288, 1998.

Gottlieb G, Corcos D, Agarwal G. 1989. "Strategies for the control of single mechanical degree of freedom voluntary movements. Behavioral and Brain Sci.” 12(2):189-210.

Gottlieb G, Song Q, Hong D, Almeida G, Corcos D. 1996. "Coordinating movement at two joints: a principle of linear covariance." Neuro phys. 75(5):1760-1764.

Hashimoto S, Narita S, Kasahara H, Shirai K, Kobayashi T, Takanishi A, Sugano S, Yamaguchi J, Sawada H, Takanobu $\mathrm{H}$, et al. 2002. "Humanoid robots in waseda university hadaly 2 and wabian." Auto Robot. 12:25-38.

Hirai K, Hirose M, Haikawa Y, Takenaka T. 1998. "The development of honda humanoid robot." IEEE Int Conference on Robot Auto. pp. 1321-1326.
Jeanneau A, Herder J, Laliberté T, Gosselin C. 2004. "A compliant rolling contact joint and its application in a 3-dof planar parallel mechanism with kinematic analysis." In Proc. DETC 2004 Salt Lake City, Utah.

Ker R, Bennett M, Bibby S, Kerster R, Alexander RM. 1987. "The spring in the arc of the human foot." Nature, 325:147-149.

Kiriazov P. 1991. "Humanoid robots: How to achieve human-like motion." J Biomech. (24):21-35.

Koopman B, van der Helm F, Veltink P. 2001. Human motion control. University of Twente, Enschede; University of Technology, Delft.

Kuo A. 1998. "Energetics of actively powered locomotion using the simplest walking model." ASME J Biomech Eng. 124:281-288.

Kuo A. 1999. "Stabilization of lateral motion in passive dynamic walking." Int J Robot Res. 18(9):917-930.

Kwek L, Wong E, Loo C, Rao M. 2003. "Application of active force control and iterative learning in a 5-link biped robot." Journal of Intelligent and Robotic Systems, 37(2):143-162.

Latash M, Gottlieb G. 1991. "An equilibrium-point model for fast single-joint movement. similarity of single-joint isometric and isotonic descending commands." J Motor Behavior. 23:163191.

Lim H-O, Setiawan S, Takanishi A. 2004. "Position-based impedance control of a biped humanoid robot." Adv Robot. 18(4):415-435.

Maloiy G, Heglund N, Prager L, Cavagna G, Taylor C. 1986. "Energetic costs of carrying loads: have african women discovered an economic way." Nature. 319:668-669.

McGeer T. 1990. "Passive walking with knees." IEEE Int Conference on Robot and Auto. 2:1640-1645.

McGeer T. 1990. "Passive dynamic walking." The Int J Robot Res. $9(2): 62-82$.

McIntyre J, Bizzi E. 1993. "Servo hypotheses for biological control of movement." J Motor Behavior 25(3):193-202.

McMahon T. 1984. "Mechanics of locomotion. Int J Robot Res." $3(2): 4-28$.

Paluska D, 2000: Design of a humanoid biped for walking research. Master's engineering department: MIT, Massachusetts; Cambridge.

Pinter M, Dimitrijevic M, Gerasimenko Y. 1998. "Effect of reduced afferent input on lumbar cpg in spinal cord injury subjects." Society Neuro. 24:623-624.

Pratt G, Williamson M. 1995. "Series elastic actuators." IEEE Int Conferences on Intelligent Robot and Sys. (1):399406.

Pratt J, Chew C, Torres A, Dilworth P, Pratt G. 2001. "Virtual model control: An intuitive approach for bipedal locomotion." nt J Robot Res. 20(2):129-143.

Sardain P, Rostami M, Thomas E, Bessonnet G. 1999. "Biped robots: Correlations between technological design and dynamic behaviour." Control Eng Practice 7:401-411.

Saunders J, Inman V, Eberhart H. 1953. "The major determinants in normal and pathological gait." J Bone and Joint Surg. 35A:543-558.

Scarfogliero U, Folgheraiter M, Gini G. 2004. “Advanced steps in biped robotics: Innovative design and intuitive control through spring-damper actuator.” In Proc. IEEE Humanoids 2004, Los Angeles, USA.

Scarfogliero U, Folgheraiter M, Gini G. 2004. "Larp: Biped robotics conceived as human modelling." In Proc. Neurobotics KI 2004, Ulm, Germany.

Scarfogliero U, Folgheraiter M, Gini G. Larp: Biped robotics conceived as human modelling. Springer LNAI (edited Wernter, Palm, Elshaw) 3575:299-314. 
Sellaouti R, Stasse O, Kajita S, Yokoi K, Kheddar A. 2006. "Faster and smoother walking of humanoid hrp-2 with passive toe joints." IEEE International Conference on Intelligent Robots and Systems, IROS.

Surla D, Borovac B, Vukobratovic M. 1989. "An approach to biped control synthesis. Robot." 7:231-241.

Vaughan C. 1996. "Are joint torques the holy grail of human gait analysis." Human Movement Sci. 15:423-443.

Vaughan C. 2003. "Theories of bipedal walking: an odyssey." J Biomech. 36:513-523.
Wisse M, Frankenhuyzen Jv. 2002. "Design and construction of mike; a $2 \mathrm{~d}$ autonomous biped based on passive dynamic walking." Delft University of technology.

Wisse M, Schwab A, Linde R. 2001. "A 3d passive dynamic biped with yaw and roll compensation." Robot. (19)275284.

Yamaguchi J, Takanishi A. 1997. "Design of biped walking robot having antagonistic driven joint using nonlinear spring mechanism." IROS 97, 251259 . 

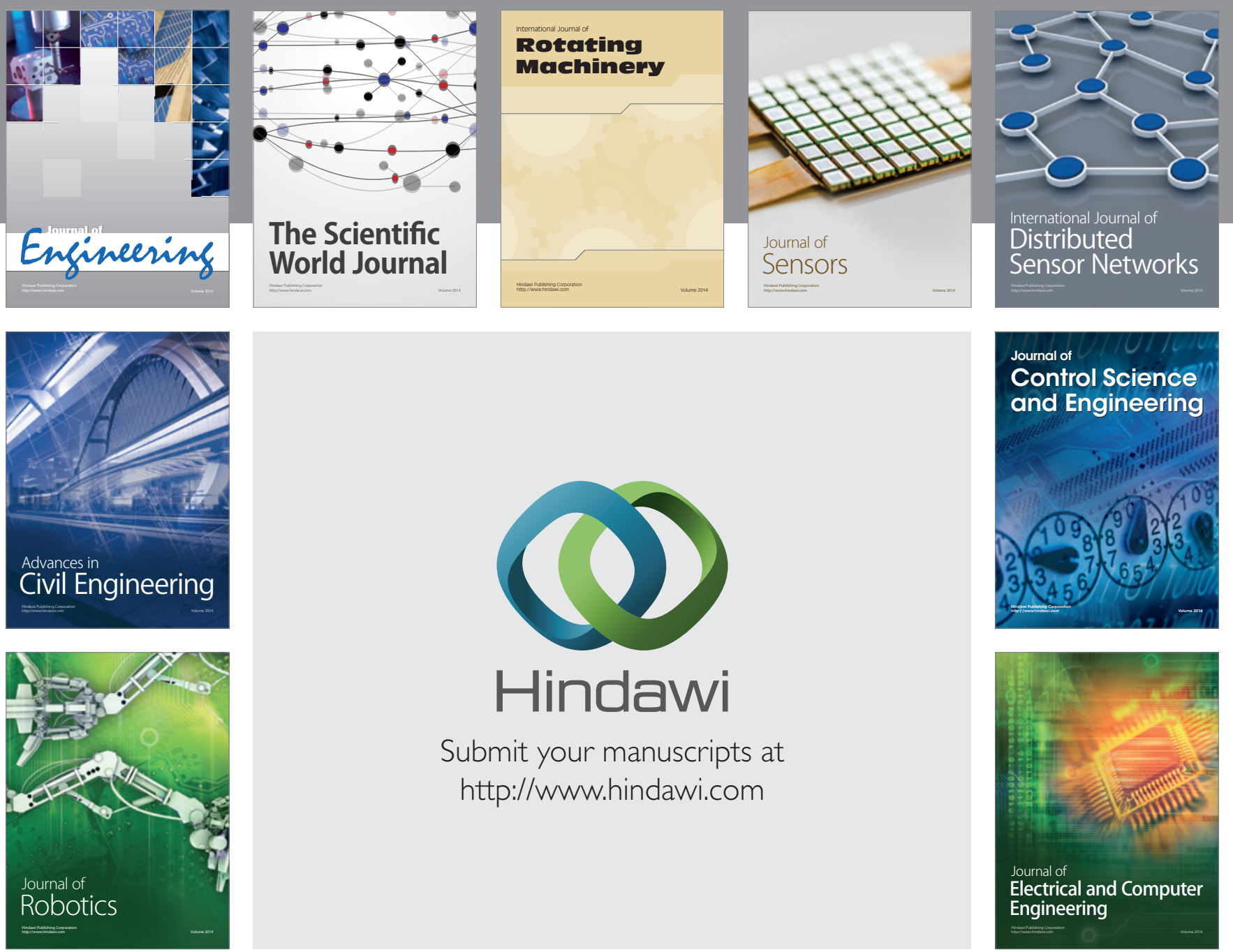

Submit your manuscripts at

http://www.hindawi.com
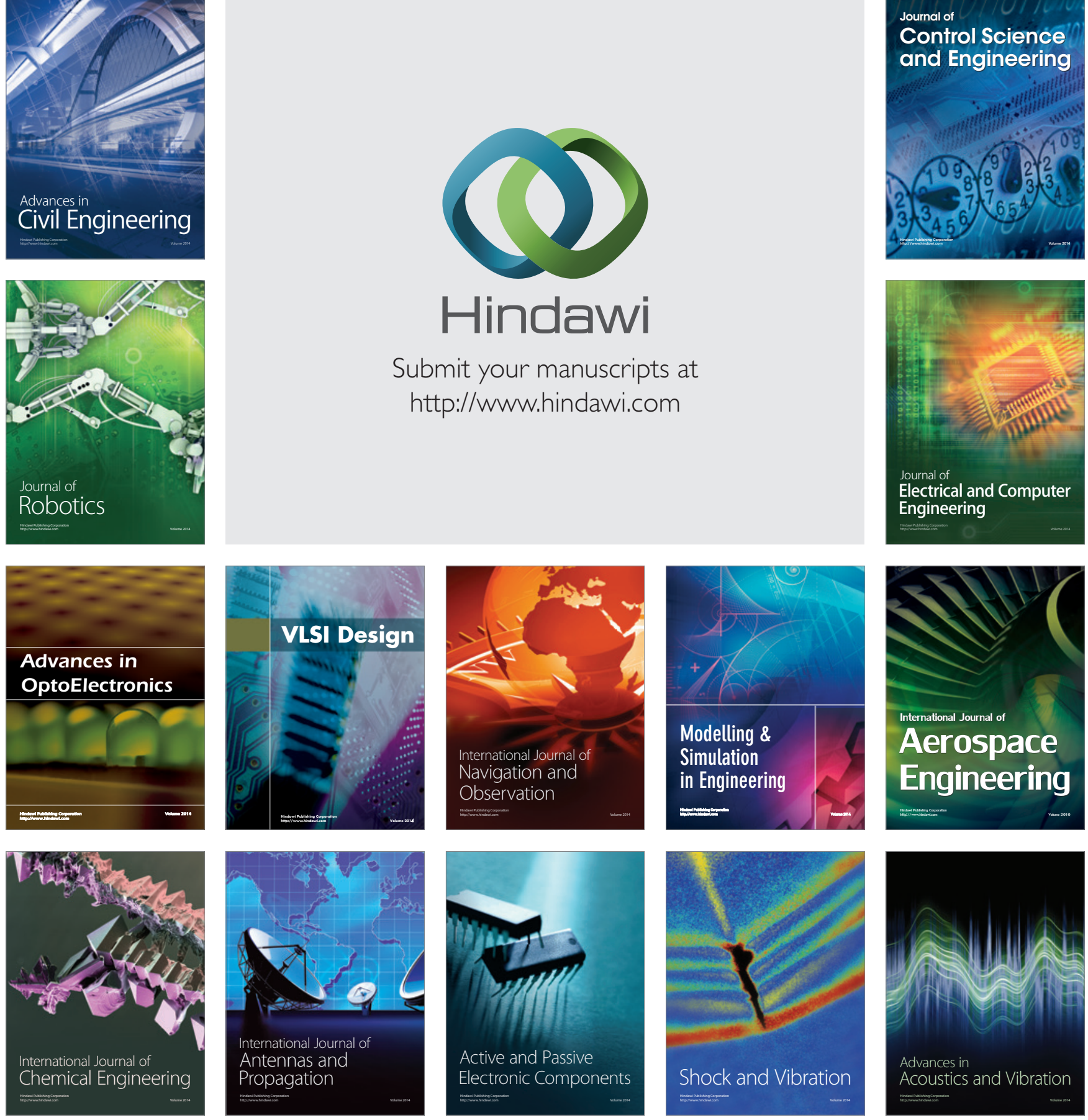